\title{
Más allá del concilio. El catolicismo holandés, un laboratorio de renovación eclesial $(1960-1975)^{*}$
}

Beyond the council. Dutch Catholicism, a laboratory for church renewal (1960-1975)

\section{Lodewijk WINKELER}

Katholiek Documentatie Centrum, Radboud Universiteit, Nijmegen

L.Winkeler@kdc.ru.nl

\begin{abstract}
Over the course of the conciliar years, progressive social movements arose in Dutch society. These were marked by the spread of youth culture and «flower power» as well as student demonstrations and the radicalization of trade unions. In the Catholic Church, this transformation was reflected in the emergence of grassroots communities and «critical parishes»). Their main concerns were: the modernization of the liturgy, social critique and protest targeting the way the Church operated, particularly concerning ecclesiastical celibacy and the obstacles to intercommunion between Catholics and Protestants. Widely covered in the media, particularly thanks to the efforts of the Septuagint, a group of protesting priests, the experience of the Dutch Catholic left was closely observed abroad in the late 1960 s and early 1970 . It subsequently lost its social basis and rapidly declined.
\end{abstract}

Keywords: Netherlands; Catholicism; Church Criticism; Grassroots Communities; Celibacy.
Resumen: Durante los años conciliares, la evolución de la sociedad holandesa hacia la izquierda está marcada por el desarrollo de una cultura joven y de flower power, pero también por las manifestaciones estudiantiles y por un endurecimiento de la acción sindical. Este cambio se traduce en la Iglesia católica en la aparición de comunidades de base y parroquias críticas. Los ejemplos analizados en el artículo, ponen de manifiesto sus preocupaciones: modernización de la liturgia pero también crítica de la sociedad y contestación del funcionamiento eclesial -particularmente el celibato eclesiástico y los obstáculos puesto a la intercomunión entre católicos y protestantes-. Ampliamente mediatizada, especialmente a través del grupo de sacerdotes contestatarios, la Septuaginta, la experiencia de izquierdas del catolicismo holandés tuvo un gran eco internacional alrededor de los años 60-70, antes de perder su base social y declinar rápidamente.

Palabras clave: Países Bajos; catolicismo; crítica a la Iglesia; comunidades de base; celibato.

* Este texto apareció, por primera vez, en Lodewijk WINKELER, Au-delà du concile : le catholicisme néerlandais, un laboratoire de renouveau ecclésial (1960-1975), Histoire@Politique, [en ligne], n 30 (septembre-décembre 2016), www.histoire-politique.fr. Agradecemos a la revista Histoire@Politique su permiso para traducirlo y reproducirlo. 


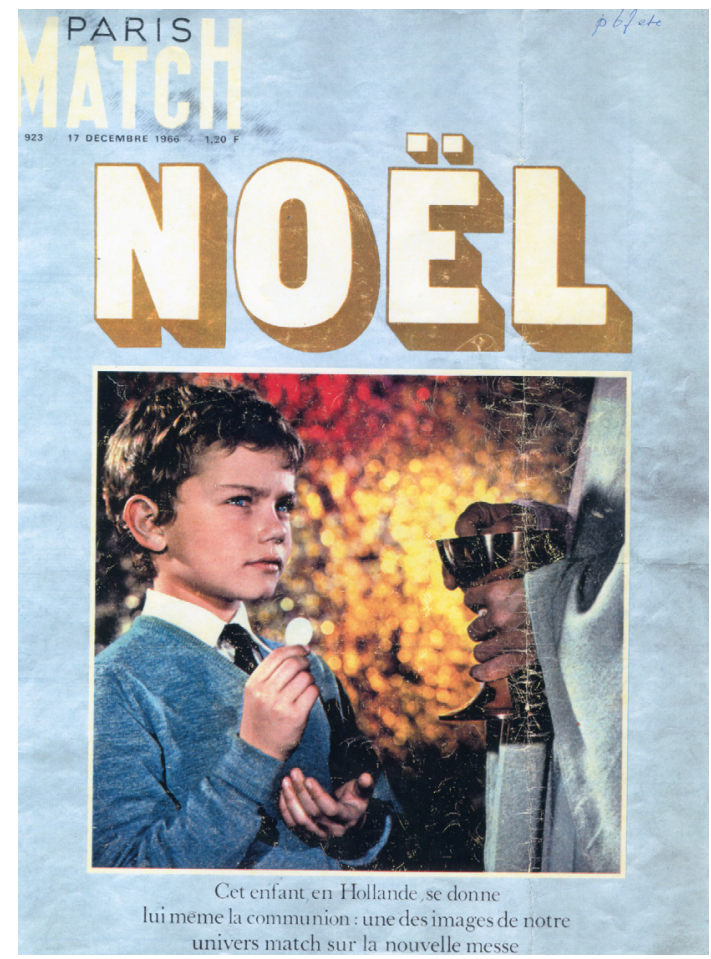

Portada de la revista Paris Match, navidades de 1966. (C) Coll. Katholiek Documentatie Centrum (KDC) en Nimega (1a32718) todos los derechos reservados.

En la portada de su número especial de Navidad de 1966, Paris Match publicaba una foto que resumía ella sola el estado del catolicismo en los Países Bajos de la época: mostraba un niño que recibía la comunión, no en la lengua, según el uso acostumbrado, si no en la mano. Los católicos holandeses aparecían en los periódicos de todo el mundo: apenas había terminado el Concilio Vaticano II y daba la impresión de que en los Países Bajos ya estaba permitido cambiar todas las cosas. Un conocido periodista, Michelle van der Plas, intentó con la publicación en 1967 de su libro Those Dutch Catholics explicar al mundo el significado de esos cambios $^{1}$, pero el mal ya estaba hecho; a los ojos del Vaticano, los Países Bajos se

1 Michel van DER Plas y Henk SuÈr (dirs.), Those Dutch Catholics, Chapman, Londres, 1967. 
habían convertido en la oveja perdida que debía volver al redil. A partir de 1970, el Papa nombró sobre todo obispos conservadores, es decir muy conservadores, para llevar a cabo las reformas necesarias. Los conflictos entre progresistas y conservadores en el seno de la comunidad católica se exacerbaron, hasta el punto de sacudir los cimientos de la Iglesia hasta los años $90^{2}$.

\section{PREHISTORIA}

Como otros países de Europa Occidental, los Países Bajos conocieron el surgimiento, justo después de la Segunda Guerra Mundial, de Corrientes progresistas situadas políticamente a la izquierda e interconfesionales. De esta manera, apareció un movimiento reformador, el movimiento de apertura (Doorbraakbeweging), que se inspiraba en el pensamiento de Emmanuel Mounier. En los Países Bajos, el personalismo ha sido retomado, en efecto, a la vez tanto por los socialdemócratas cómo por el movimiento ecuménico. El Partido Social Demócrata de los trabajadores (SDAP) de antes de la guerra dio lugar al nacimiento, después de la guerra, del Partido del Trabajo (PvdA), también de orientación social-demócrata, que tenía como vocación acoger adherentes de todas las confesiones religiosas. Al lado de los líderes socialistas, los pastores protestantes liberales y un cierto número de intelectuales católicos contribuyeron a elaborar la doctrina del partido. Pero los obispos se opusieron a esta evolución con una gran eficacia: a partir de 1944, cuando el norte del país estaba aún ocupado por los alemanes, decidieron restablecer «sin más dilación» el conjunto de organizaciones católicas, e incluso el partido de Estado católico romano, rebautizado como Partido Popular católico (KVP). A pesar de este retorno a las organizaciones de antes de la guerra, la ideología progresista se había anclado en el catolicismo holandés, especialmente en el seno de la comunidad católica del trabajo (Katholieke Werkgemeenschap), un organismo afiliado al Partido del Trabajo, y en el periódico Te Elfder Ure (La undécima hora). Está publicación había sido fundada dentro de la Resistencia bajo el nombre de Christoffor ${ }^{3}$.

2 Un resumen de la historia religiosa de los Países Bajos después de la guerra, en George HARINCK y Lodewijk Winkeler, The Twentieth Century, en Herman J. SELDERHUIS (dir.), Handbook of Dutch Church History, Vandenhoeck \& Ruprecht, Göttingen, 2015, pp. 566-644.

3 Gerard DIERICK, De Christofoorgroep en het tijdschrift Te Elfder Ure in de jaren 1945-1953, en Faarboek van het Katholiek Documentatie Centrum, 13 (1983), pp. 35-90; Fons D'HAENS, De Katholieke Werkgemeenschap in de Partij van de Arbeid en de politieke doorbraak, en faarboek van het Katholiek Documentatie Centrum, 4 (1974), pp. 59-98. 
En 1953, el sacerdote Simón Jelsma, m.s.c., lanzó el movimiento Pleingroep (Grupo de la plaza), compuesto de católicos que deseaban abrir la Iglesia al mundo moderno. Jelsma se dio a conocer por sus sermones en la plaza pública (Pleinpreken) de La Haya. Esta corriente de pensamiento había llegado a ser lo suficientemente influyente como para que los obispos holandeses se sintieran obligados a publicar, en 1954, un mandato exhortando a los católicos a preservar su unidad. Según esta indicación, estaba prohibido a los católicos escuchar la radio socialista (VARA), ser miembro de un sindicato socialista o adherirse a la Asociación holandesa para la reforma sexual (dónde se podían comprar contraceptivos). La adhesión al Partido del Trabajo no estaba formalmente prohibida, pero los católicos que se arriesgaban sufrían una fuerte presión de parte de la comunidad a fin de que renunciaran, por otra parte, con poco éxito. «Se trata de una dictadura, pero basada en la Palabra de Dios», comentaba un sacerdote, como reacción al mandato episcopal ${ }^{4}$. De hecho, este mandato provocó un efecto boomerang. A partir de 1957, la Asociación San Willibrordo, una prestigiosa asociación, con decenas de miles de miembros, que militaba en favor de las relaciones ecuménicas, público el informe Apertura y cerrazón en los católicos holandeses, que criticaba fuertemente el mandato Episcopal ${ }^{5}$. Dos años más tarde, Juan XXIII anunciaba la celebración del Concilio Vaticano II.

\section{Los Países Bajos y el Concilio VaTiCANO II (1962-1965)}

Cómo en todas partes, el anuncio del Concilio fue recibido con prudencia $^{6}$. Los documentos de trabajo elaborados por la curia romana proponían pocos avances. El entusiasmo no empezó a manifestarse en los Países Bajos -en los Países Bajos y en todo el mundo-, hasta que los padres conciliares no tuvieron la certeza de poder ir más allá de los esquemas preparatorios. Entre los textos votados por el Concilio, era sobre todo la Constitución pastoral Gaudium et spes, que trataba sobre el papel de la Iglesia en el mundo moderno (1965), la que marcó los

4 Jan BANK, «Dictatuur, maar gebaseerd op Gods gezag». Het bisschoppelijk mandement van 1954, en Intermediair, 15 (1979), nr. 20, pp. 1, 3, 5, 7, 9, 11-12; Jan HINKE, Het bisschoppelijk mandement van 1954 in pers en politiek, mei 1954-februari 1955, en Jaarboek van het Katholiek Documentatie Centrum, 19 (1979), pp. 73-116.

5 Cfr. Jan JaCOBS, Nieuwe visies op een oud visioen. Een portret van de Sint Willibrord Vereniging, 1948 1998, Valkhof Pers, Nijmegen, 1998, pp. 77-82.

6 ID., Met het oog op een andere kerk. Katholiek Nederland en de voorbereiding van het Tweede Vaticaans Oecumenisch Concilie 1959-1962, Nelissen, Baarn, 1986. 
espíritus. Los teólogos holandeses llegados a Roma como expertos tuvieron un gran papel en la mayoría conciliar, influyendo no solo en los obispos holandeses, sino también en algunos homólogos extranjeros ${ }^{7}$. El teólogo flamenco Edward Schillebeeckx, dominicano, que trabajaba en los Países Bajos, alcanzó una notoriedad internacional.

En los Países Bajos, el aggiornamento del Concilio fue muy seguido e incluso anticipado. En el campo de la liturgia, las facilidades que la encíclica Mediator Dei (1947) había autorizado, sobre todo el uso, aún limitado, de la lengua vernácula, fueron adoptadas en todas partes. En las parroquias, se formaron numerosos grupos de discusión desde inicios de los años 60 y, entre ellos, grupos de espíritu ecuménico. En 1971, el país tenía algo más de 5000 grupos de discusión con un número de participantes que se elevaba hasta las 55000 personas en total: estás cifras daban como resultado casi tres grupos por cada parroquia. En esos grupos se expresaban abiertamente las dudas respecto a las tradiciones religiosas y el papel de la Iglesia dentro de la sociedad.

Al mismo tiempo, la generación del baby boom, nacida después de la Segunda Guerra Mundial, empezaba a hacerse escuchar. Está generación criticaba los valores tradicionales de la generación precedente, y un movimiento pintoresco, anti burgués y orientado a la izquierda, se empezaba a desarrollar. Dentro de la sociedad, se manifestó en la aparición de la cultura joven y en la idea del flower power, pero también se expresaba a través de manifestaciones estudiantiles, de la radicalización de los movimientos sindicales (especialmente de los jóvenes obreros) y en la crítica a los Estados Unidos en general y a la guerra del Vietnam en particular ${ }^{8}$. El empuje progresista no se limitaba a la juventud y a los estudiantes. La sociedad holandesa, en su conjunto, giró a la izquierda, y las organizaciones católicas no fueron una excepción a esta regla. El Partido Popular católico (KVP) publicó en 1966 su nuevo estatuto: Fundamento y carácter del KVP. A partir de ese momento, el partido ya no pretendía ser la expresión de la doctrina católica en política y se declaraba libre del control de los obispos. El partido decidía dejarse «orientar e inspirar por la visión cristiana sobre los hombres del mundo, tal como es vivida en el seno de la comunidad de la Iglesia católica»${ }^{9}$ Pero para algunos

7 Jan BROUWERS, Vreugde en hoopvolle verwachting. Vaticanum II, terugblik van een ooggetuige, Arbor, Baarn, 1989.

8 Para una presentación detallada, véase James C. KENNEDY, Nieuw Babylon in aanbouw. Nederland in de jaren zestig, Boom, Amsterdam, 1995.

9 Hans BorneWASSER, Katholieke Volkspartij 1945-1980, tome II, Valkhof Pers, Nijmegen, 2000, pp. 31-34. 
de sus miembros, la política del KVP no era aun suficientemente progresista. Dos años más tarde, una escisión llevará a la fundación del Partido político de los radicales (PPR).

Alfons Arnolds, director de la oficina científica del Movimiento sindical católico (NKV), constató en 1968, en su Informe sobre la confesionalidad del NKV, que un movimiento sindical no tenía necesidad de ser confesional para asegurar «una transmisión fructífera y eficaz del mensaje evangélico de humanidad, de justicia y de amor en la sociedad, en la vida económica y en sus estructuras ${ }^{10}$. Ocho años más tarde, el NKV se fusionaba con el Agrupamiento holandés de asociaciones sindicales, de orientación socialista, al cual estaba prohibida, dos decenios antes, la adhesión de los católicos por la Iglesia. La Organización de la radio católica (KRO) evolucionó también hacia la izquierda, bajo la dirección de Harry van Doorn, uno de los cofundadores del PPR, y público, en 1969, un informe muy progresista, izquierdista, titulado KRO. El futuro como desafí ${ }^{11}$.

\section{LAS COMUNIDADES DE BASE Y LAS PARROQUIAS CRÍTICAS}

Entre 1975 y 1977, el grupo de trabajo sobre las comunidades de base y las parroquias críticas en los Países Bajos había efectuado un recuento, en base a una encuesta, de las nuevas formas de agrupación y del número de sus miembros ${ }^{12}$. En aquel entonces, contaba con poco más de 80 comunidades, muy diversas en su composición: católicas, protestantes o ecuménicas. Unas se concentraban en la crítica a la sociedad, las otras en la reforma litúrgica; algunas estaban ligadas a una comunidad religiosa, otras eran independientes. Me limitaré, en un primer momento, a cuestiones generales. Luego entraré en los detalles interesándome en algunos casos típicos.

Lo que llama la atención en primer lugar, es que los grupos de base tienen dos características comunes. Primero su composición social, que se nutre masivamente de las clases medias y de los intelectuales, cosa que no es sorprendente porque lo mismo se observa en la casi totalidad de los movimientos de

10 Jan PEET, Tussen aanpassing en vernieuwing. Hoofdlijnen in de geschiedenis van het Nederlands Katholiek Vakverbond, 1963-1981, en Jan RoEs (dir.), Katbolieke arbeidersbeweging, Ambo, Baarn, 1985, p. 141.

11 Ad MANNING, Zestig jaar KRO. Uit de geschiedenis van en omroep, Ambo, Baarn, 1985, pp. 265-275.

12 Gerard DieriCK, Basisgroepen in Nederland. Signalementen, Katholiek Documentatie Centrum, Nijmegen, 1977. El análisis de esta documentación ha sido hecho por mi para preparar este artículo. 


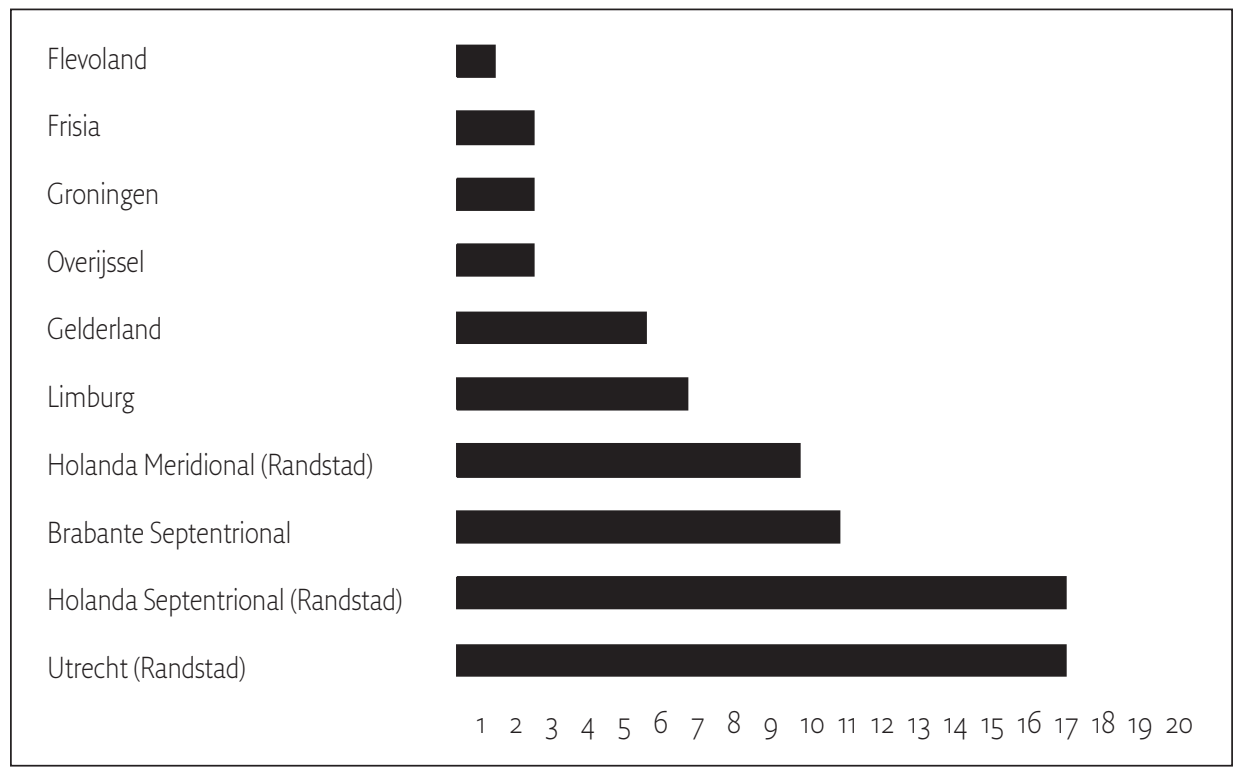

Gráfico 1. Comunidades de base y parroquias críticas por provincia.

contestación de izquierdas. Muchos de sus participantes habían tomado parte, con anterioridad, en los grupos de discusión católica mencionados más arriba. En la encuesta, algunos grupos de base hace mención explícita a esta ascendencia. En realidad, algunos de estos grupos de discusión simplemente han seguido a su actividad bajo otro nombre, el de «grupo de base». Una segunda característica común a estos grupos es su orientación política, casi sin excepción de izquierdas, es decir muy de izquierdas. Lo que no implica, a la fuerza, que todos los grupos militaran activa y públicamente en el ámbito político, social o eclesial. En muchos casos, su crítica se quedaba en la protesta verbal e interna.

Algo que atrae la atención es que la mayor parte de esos 43 grupos de base, y en particular los más críticos, estaban localizados en las provincias más urbanizadas: Utrecht, Holanda-septentrional, Holanda-Meridional (lo que se llama «el Randstad»). Dieciséis de ellos habían surgido en ciudades universitarias y otros cuatro se encontraban en ciudades universitarias exteriores al Randstad. Estaban establecidos en las provincias de tradición católica, Brabante Septentrional y Limburg, pero la mayor parte, habían surgido en comunidades residenciales o de los grupos que se habían constituido alrededor de una celebración alternativa de la misa. 


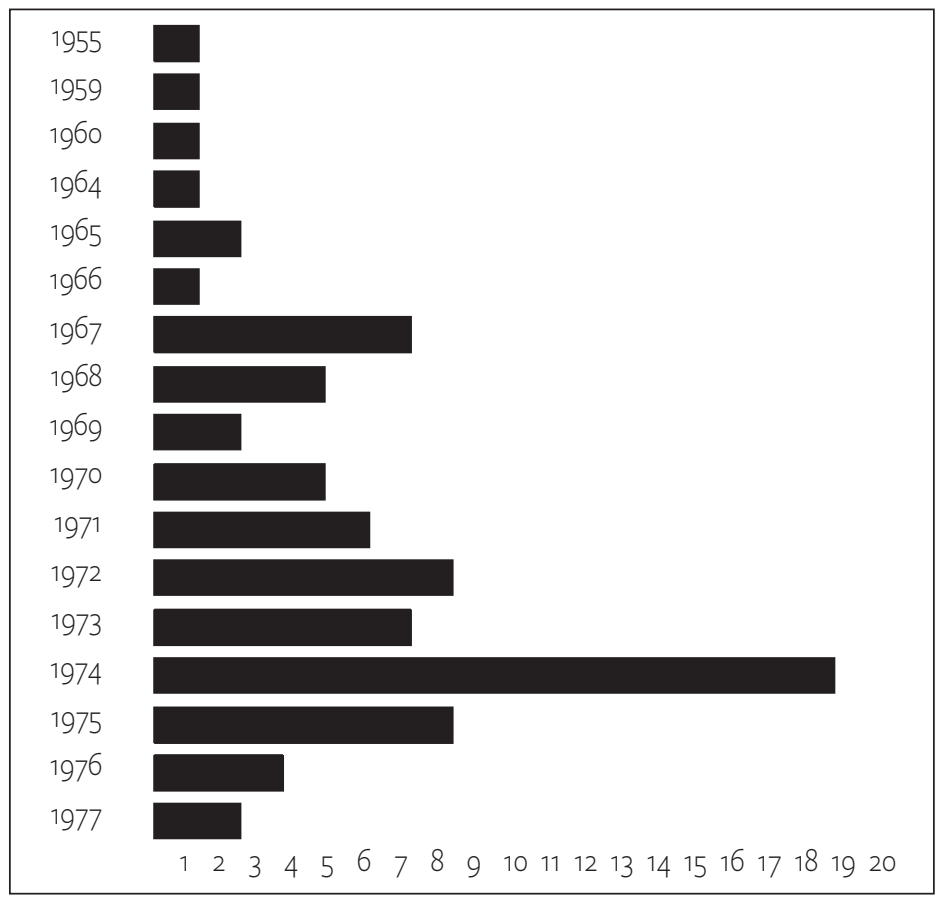

Gráfico 2. Comunidades de base y parroquias críticas, año fundación.

A pesar de los puntos en común, las diferencias son aún más numerosas. He excluido de mi análisis los diecisiete grupos protestantes y los dos grupos no religiosos. Mi base de datos comprende entonces 69 grupos, de los cuales 37 son ecuménicos y 32 católicos en sí mismos, aunque entre estos últimos, no todos se llaman católicos por principio, pero sí en su origen. Igualmente me he interesado en su año de fundación. La mayor parte han sido fundados después de 1967, pero sobre todo después de 1970, y estos últimos han sido más críticos en su aproximación que sus predecesores. La explicación de este fenómeno es sencilla: a partir de 1969-1970, los obispos se encontraban en una situación difícil cara a Roma en razón de la petición de la abolición del celibato sacerdotal por parte de algunos católicos holandeses. El episcopado se había visto obligado a reforzar la disciplina, con más razón puesto que fieles conservadores denunciaban directamente a la Santa Sede las violaciones de las normas eclesiásticas. De allí que la aparición de conflictos entre diferentes grupos de base y los obispos, haya sido la causa de la radicalización de las posturas. 


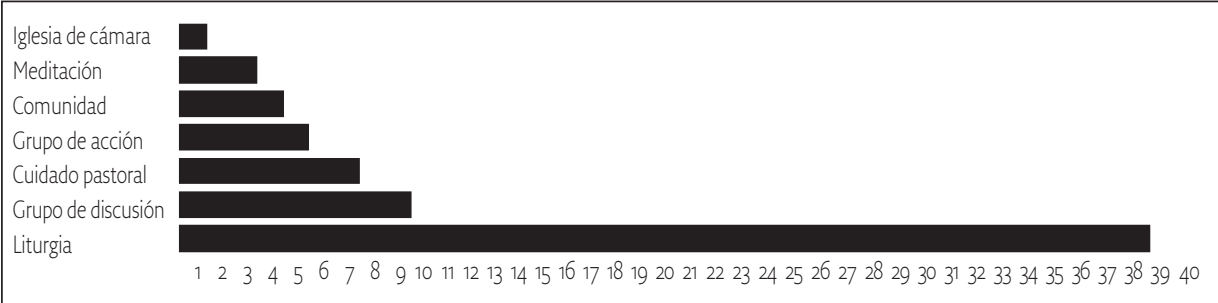

Gráfico 3. Comunidades de base y parroquias críticas, actividad principal.

Disponemos de muy poca información sobre la desaparición de estos grupos. Sobre el número de sesenta y nueve, cinco de ellos habían ya dejado de existir en el momento del inventario. Muchos de ellos desaparecieron en razón del envejecimiento de sus miembros, de la pérdida de interés por las actividades comunes o por las dificultades financieras. En lo que respecta a las actividades, parece que 38 grupos se habían constituido alrededor de la celebración dominical. En la otra parte del espectro, encontramos cinco grupos que han dado la prioridad a la crítica social. Entre los dos, hay coincidencias en hechos muy diversos: discusión, lugar de residencia, animación pastoral, la mayor parte del tiempo a cargo de estudiantes.

También podemos estudiar estos grupos a partir de la descripción que ellos mismos hacen en su respuesta a la encuesta. Entre estos grupos, veintiuno invocan el ecumenismo, veinte la contestación eclesial, veintitrés la crítica social, pero aun así sabemos que muchos de ellos no estaban dispuestos a la acción. En resumen, la mayor parte de ellos están orientados hacia la izquierda y adoptaban una actitud crítica frente a la Iglesia sin hacer de ello una cuestión de principio. Estos grupos desarrollaban sus actividades, pero evitando hacerlas públicas. Un ejemplo: no solamente en el seno de los grupos ecuménicos, sino también en los mayoritariamente compuestos de católicos, la inter comunión era una práctica habitual. Era necesario, sin embargo, evitar hablar de eso demasiado abiertamente. En cuanto los efectivos, las estadísticas poco sirven para establecer su número. Disponemos de indicios sobre el número de participantes y de simpatizantes, pero hay grandes disparidades. Los grupos realmente militantes contaban generalmente entre 10 y 50 miembros. Las parroquias de estudiantes, por el contrario, podían llegar a convocar entre 500 y 2000 fieles todos los domingos. Se puede estimar la audiencia global todos esos grupos en alrededor de 20000 personas. 


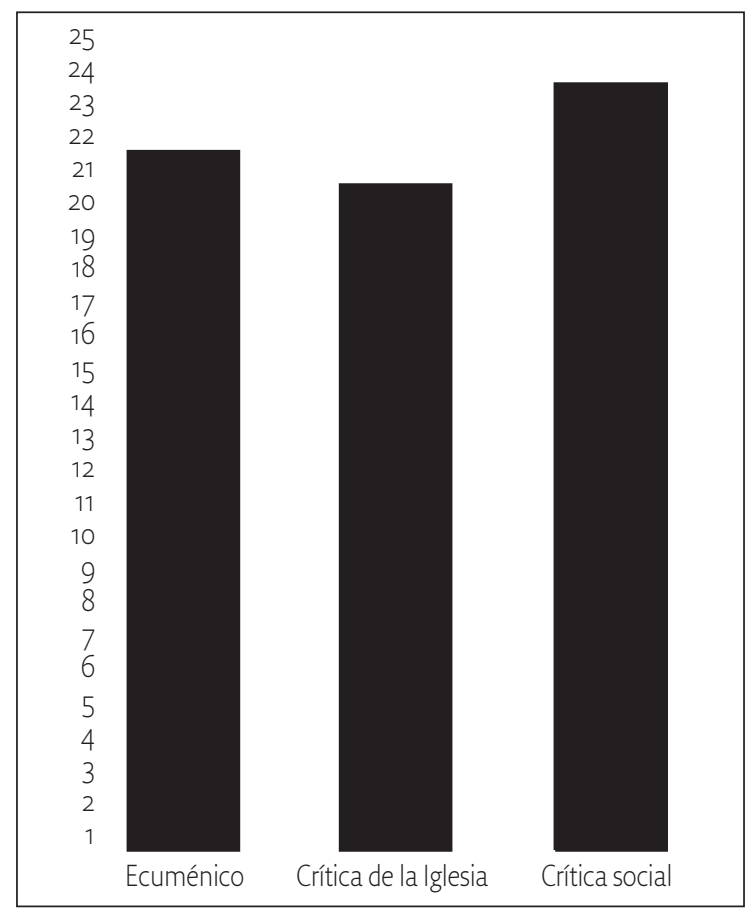

Gráfico 4. Comunidades de base y parroquias críticas, autoimagen.

\section{CINCO EJEMPLOS}

Las experiencias descritas hasta ahora permiten ilustrar los elementos característicos de los grupos de base o de las parroquias críticas: modernización de la liturgia, contestación social, conflictos intraeclesiales, especialmente en lo que respecta a la intercomunión y al celibato sacerdotal.

\section{El grupo Sjaloom}

Se trata del Pleingroep, que se había formado en La Haya alrededor del sacerdote Simón Jelsma, que ha alumbrado una de las primeras comunidades de base de los Países Bajos, el grupo Sjaloom ${ }^{13}$. En 1963, el Consejo de la juventud ecu-

13 Gerard VAN TILlO, «Sjaloom». Een sociologische oriëntatie op de veranderingen in de religie van een sociaal-politiek actiegroep, en G. SCHIPPER-PEET et al. (dir.), Tussen hemel en aarde. Beschouwingen over bedendaagse religieuze bewegingen, Samson, Alphen aan de Rijn, 1976, pp. 208-246. 
ménica de los Países Bajos organizó el congreso ecuménico juvenil, «Cinco panes y dos peces», reuniendo a 5000 jóvenes. Entre las organizaciones participantes se encontraba igualmente el Pleingroep. El grupo de trabajo que estaba en el origen de este encuentro había sido el primer núcleo del Sjaloom, que se había ampliado para formar rápidamente un grupo de una treintena de miembros de las diferentes iglesias. Entre ellos, Jelsma y su primo segundo Piet Reckman, profesor en la Academia social De Horst (orientada hacia la izquierda) e ideólogo del grupo. En 1965, Sjaloom se muda a Odijk, un pueblo en el centro de Holanda. Junto a las reuniones de estudio que reunían a un gran número de participantes, los grupos practicaban también en reuniones ecuménicas, llamadas de agapé, con intercomunión:

La celebración del agapé es una comida, durante la cual se habla, se fuma y otras cosas por el estilo. No se leen solamente pasajes de la Biblia, sino también textos sacados de periódicos y revistas. Se dice el Credo y oraciones. En el momento de la comunión, se parte el pan recíprocamente dando gracias y, una vez la copa de cada uno está llena, se brinda deseándose Sjaloom.

El grupo reúne en esta época cerca de 300 personas. Al final de los años 60, Sjaloom amplía su significado a oikumenè ya identificado con el mundo entero. Pendiente de las Conferencias de las Naciones Unidas sobre el comercio y el desarrollo de 1964, 1968 y 1972, se moviliza cada vez más en el ámbito del tercermundismo. En los inicios de los años 70, las celebraciones dominicales se parecen cada vez más a sesiones de formación política de izquierdas: todo gira en torno al «análisis de un sistema cultural, en el cual se trata de descubrir los mecanismos que esconden las verdaderas desigualdades y que mantienen una falsa conciencia» ${ }^{14}$. Es en esta época cuando Piet Reckman recibe el apodo de «el Lenin de Odijk». Sjaloom, que había comenzado su andadura en el mundo ecuménico, se había convertido progresivamente en un movimiento de crítica social antes de desaparecer en 1976.

\section{La Iglesia de jóvenes de Venlo}

Un caso bien diferente, es la Iglesia de los jóvenes de Venlo, fundada, como su nombre indica, en la villa limburguesa de Venlo, y que hoy día sigue funcionando ${ }^{15}$.

14 VAN TILlo, Sjaloom..., op. cit., p. 224.

15 Véase Harry Notenboom, Alles heeft zijn tijd. Fongerenkerk Venlo, Van Spijk, Venlo/Antwerpen, 1998. 
Está iglesia tiene su origen en las conferencias de Cuaresma organizadas a partir de 1958 por el sacerdote Leo Brueren, para y en colaboración con los jóvenes de Venlo. Estos se aburrían muchísimo en la misa dominical. Como otros, querían misas dirigidas a ellos mismos y una coral propia dónde cantar los cánticos compuestos por ellos sobre melodías de tipo góspel. La parroquia puso entonces a su disposición una iglesia en desuso y, a partir de 1965, la Iglesia de jóvenes atrae todos los domingos más de un millar de quinceañeros. $\mathrm{Al}$ margen de estas celebraciones, se crean grupos de discusión. Los obispos del tercer mundo, de paso por Europa, son invitados a celebrar la misa y es de esta manera que se desarrolla el compromiso social de la Iglesia de jóvenes, que sostiene asociaciones de ayuda a los sin hogar y a los refugiados.

Dentro de este contexto, el nombramiento del obispo conservador Joannes Gijsen, en 1972, supuso un shock. El equipo dirigente de la Iglesia de los jóvenes se prepara para un enfrentamiento abierto, pero el obispo les deja tranquilos, mientras que, por otra parte, lleva a cabo una política dura frente a los que dependen de él. Esta actitud, probablemente, es debida al apoyo tácito del vicario general, conservador como él, pero antiguo camarada de estudios de Brueren. En el curso de los años setenta, la asistencia disminuye. Los jóvenes que habían estado allí desde sus orígenes, convertidos ya en padres, continúan viniendo, en primer lugar, con sus hijos y más tarde sin ellos. Hoy día, la Iglesia de jóvenes de Venlo cuenta con centenares de simpatizantes ${ }^{16}$. Su orientación ha sido siempre la de una comunidad moderada, preocupada sobre todo de una celebración atrayente de la misa dominical. A excepción de la puesta en escena litúrgica, siempre ha respetado las reglas impuestas por la iglesia.

\section{La parroquia estudiantil de Utrecht}

Tercer ejemplo. La parroquia estudiantil de Utrecht había sido fundada durante la Segunda Guerra mundial. En esa época, la universidad estaba cerrada y los estudiantes, requeridos para el servicio de trabajo obligatorio, partían hacia Alemania o entraban en la clandestinidad. Para mantener el contacto, se fundaron las parroquias de estudiantes, ya que los alemanes no podían tocar a las instituciones religiosas ${ }^{17}$. Después de la guerra, la parroquia estudiantil de Utrecht

16 www.jkvenlo.nl, consultado el 14 de febrero de 2018.

17 Sobre la pastoral estudiantil en los Países Bajos, véase Lodewijk WiNKELER, Glasses Water over Petting Couples. Roman-Catholic Pastoral Care for Students in the Netherlands (1900-1980), (en prensa). 
siguió la evolución global de la Iglesia y de la sociedad, participando con optimismo en los sixties. En 1968, uno de sus cuatro capellanes, Jos Gooskens, anunció su próximo matrimonio. El Consejo de la parroquia y los tres capellanes restantes declararon que no veían ningún inconveniente en que Gooskens continuara diciendo la misa, pero el cardenal Alfrink en persona les fue a explicar que eso era contrario a las leyes eclesiásticas y que no sería así de ninguna de las maneras ${ }^{18}$. Los interesados se sometieron, pero un año más tarde estalló otro conflicto: la parroquia de estudiantes anunció que en los seis domingos de Cuaresma los capellanes protestantes celebrarían la eucaristía. A pesar de una entrevista «larga y fatigosa» con el cardenal Alfrink, que prohibía formalmente la realización de este proyecto, tuvo lugar la primera celebración. En consecuencia, los tres capellanes fueron suspendidos de sus funciones, lo que dio lugar a discusiones turbulentas en el seno de la parroquia y a la creación de una comisión de conciliación. La solución encontrada consistía en celebrar una semana la eucaristía y otra la santa cena protestante utilizando casi los mismos textos en los dos casos. Al cabo de un mes, la suspensión de los sacerdotes fue levantada. Ya no hubo más conflictos, porque la capellanía de estudiantes se interesaba cada vez menos en las cuestiones litúrgicas centrándose en los debates de la sociedad, la realización personal y la espiritualidad oriental. A partir de 1970, la Parroquia de estudiantes y la capellanía protestante establecieron una colaboración estrecha creando la Iglesia evangélica universitaria (EUG).

\section{Amsterdamse Studentenekklesia (parroquia estudiantil de Ámsterdam)}

Sin embargo, la evolución de la Parroquia de estudiantes de Ámsterdam es lo que más ha llamado la atención de la opinión pública. Su liturgia ha sido ideada, desde inicios de los años 60, por un pequeño grupo de jesuitas que aún no habían acabado sus estudios y cuyos líderes eran Huub Oosterhuis y Bernard Huijbers. Aún antes de su ordenación sacerdotal, Oosterhuis comenzó a escribir cantos litúrgicos, musicalizados por Huijbers y más tardes por Antoine Oomen ${ }^{19}$. Su influencia en los Países Bajos fue considerable. Aún hoy día, una celebración en la que no haya uno o más cantos de Oosterhuis es inimaginable. Nombrados

18 Sobre este asunto véanse los recortes de periódico en el Katholiek Documentatie Centrum Nijmegen, fondo Krantenknipsels, dosier Studentenpastoraat - Utrecht.

19 Kees KoK, De vleugels van een lied. Over de liturgische poëzie van Huub Oosterhuis, Ambo, Baarn, 1990. 
capellanes en Ámsterdam, Oosterhuis y sus compañeros concebían una litúrgica que conjugaba la estética y el compromiso político, respondiendo a las expectativas de la época.

En 1968, otro capellán, Jos Vrijburg, anunció su compromiso matrimonial. Como es lógico, la postura tomada un poco antes por el cardenal Alfrink, a propósito de la Parroquia de estudiantes de Utrecht, se mantuvo también para este caso. En 1969, Vrijburg dejó la Compañía de Jesús. Dejó de celebrar la misa, pero continuó predicando. Monseñor Theo Zwartkruis se lo prohibió, pero el equipo de la Parroquia estudiantil de Ámsterdam se resistió. Salvo una excepción, los capellanes decidieron considerarse como sacerdotes libres, no ligados a las órdenes de la jerarquía. Los cuatro rebeldes -Oosterhuis, Vrijburg, Ton van der Stap y Jan van Kilsdonk- tomaron la decisión de no atender los oficios de la parroquia de estudiantes. Las negociaciones con el episcopado se pusieron en marcha, mientras que el superior provincial y el General de los jesuitas, Pedro Arrupe, presionaron a los capellanes para que volvieran al redil. El uno de marzo de 1969, Monseñor Zwartkruis acordó un estatuto especial para la parroquia de estudiantes, bajo la condición del control por parte de una comisión ad hoc, y autorizó a Vrijburg a celebrar liturgias de la Palabra: estas se terminaron, de hecho, a los seis meses, ante la falta de interés de los fieles. Pero el General de los jesuitas no quedó contento con este compromiso: quiso expulsar a Oosterhuis y a Van der Stap de la Compañía, pero el superior provincial holandés no se prestó a colaborar en esta decisión. Finalmente, los dos interesados se marcharon por su propio pie, pero sin renunciar al sacerdocio.

Un año más tarde, Oosterhuis se casó, lo que provocó un debate dentro de la Parroquia estudiantil con motivo de las consecuencias y la idea misma del sacerdote libre y del estatuto experimental de la parroquia. El 28 de septiembre, Oosterhuis celebró públicamente la eucaristía causando un escándalo internacional. Monseñor Zwartkruis declaró que la Parroquia estudiantil ya no caía bajo su autoridad y la Compañía de Jesús puso fin al contrato de alquiler de la capilla Ignatius. De golpe, la Parroquia estudiantil se trasladó a la iglesia protestante de Amstel, siguiendo su camino como parroquia independiente y crítica. Recientemente, la comunidad ha suprimido el título de «estudiantil» de su nombre: ahora se llama Ekklesia Amsterdam ${ }^{20}$.

20 Véase VAN DijK, De paus van Amsterdam, op. cit., p. 198-386 en www.studentenekklesia.nl (consultado el 14 de febrero de 2018). 


\section{La parroquia crítica Ifmond}

La parroquia crítica IJmond, también hunde sus raíces en la renovación litúrgica de finales de los sesenta ${ }^{21}$. El joven sacerdote Jan Ruijter inaugura, en su parroquia de la ciudad industrial de Beverwijk, las misas «beat», durante las cuales un grupo musical acompaña los cantos holandeses con melodías góspel, atrayendo a un numeroso público. Poco a poco, la liturgia se carga de contenido político, expresando la visión crítica de la juventud frente a la sociedad: se evoca, por ejemplo, la paz, la guerra de Biafra o el problema de los retrasados mentales. El eco de estas iniciativas va más allá de la propia parroquia, llegando incluso a los no católicos. A principios de los 70, la Parroquia se encuentra marcada por el compromiso de sacerdotes contestatarios del movimiento de la Septuaginta, del cual Jan Ruijter era el presidente. Las celebraciones tomas cada vez más un cariz ecuménico -incluida la intercomunión- y crítico hacia la Iglesia y la sociedad. Se forma grupos de discusión en torno a los problemas de la paz, la droga, o los trabajadores inmigrantes. Esta evolución acentúa la oposición de los católicos tradicionales, si bien tanto Ruijter como sus partisanos acaban por abandonar la parroquia para organizarse libremente. En 1971, el obispo diocesano reconoce la parroquia crítica IJmond como «asociación eclesiástica».

Un nuevo paso se da cuando Ruijter se casa en 1972, pero decide continuar con su actividad sacerdotal. Él explica entonces que «mantener la obligación del celibato es un abuso de poder que testimonia el total fracaso de la Iglesia ${ }^{22}$. Como las negociaciones no llevaron a ninguna solución, el obispo dejó de reconocer la Parroquia crítica de IJmond como asociación eclesiástica. A mitad de los 70, ésta se presentaba, más bien, como un conjunto de grupos de acción social, trabajando tanto en el cuidado de las personas mayores o en el apoyo a los trabajadores inmigrantes, como en la ayuda a los discapacitados o en el albergue de personas de paso. A esta labor, se añadían las movilizaciones puntuales contra la guerra del Vietnam, o contra la dictadura chilena, por ejemplo. La parroquia crítica de IJmond existe aún hoy día, pero como una comunidad de base orientada a la reforma de la Iglesia: la dimensión política y social ya no está en el centro de sus preocupaciones ${ }^{23}$.

21 Sobre el nacimiento y los primeros años de la Parroquia crítica IJmond, véase Richard AUWERDA, Welkom en ongewenst. Vijf jaar zoeken in Beverwijk. Ontstaan van een nieuwe kerkgemeente, Gooi \& Sticht, Hilversum, 1971.

22 Richard AUWERDA, Kerk van onderen. Kritische Gemeente Ifmond 1975, Gooi \& Sticht, Hilversum, 1975. Cita p. 7.

23 Véase www.kgij.nl (consultado el 14 de febrero de 2018). 
Se percibe como la renovación litúrgica está en el origen de todos estos grupos, pero desemboca rápidamente en la crítica a la institución eclesial: la cuestión de la intercomunión, el matrimonio de los sacerdotes, o las dos cosas a la vez, llevaron a reiterados conflictos con los obispos. Algunas comunidades, como la de Utrecht, se plegaron a las reglas impuestas por la Iglesia. Otras -como la Parroquia estudiantil de Ámsterdam o la Parroquia crítica de IJmond- decidieron liberarse del control jerárquico, lo que las llevó al compromiso crítico en los campos sociales y políticos, debido a que, según su punto de vista, no había posibilidad de avanzar dentro de la Iglesia.

\section{IDEOLOGÍA DE LAS COMUNIDADES DE BASE}

Hasta aquí, me he acercado a las comunidades de base bajo el prisma de sus posturas dentro de la Iglesia, como consecuencia del movimiento de modernización salido del Vaticano II. La actitud radicalmente crítica con la sociedad, en primer lugar, por el grupo Sjaloom seguido de la Parroquia estudiantil de Ámsterdam, de la Parroquia crítica de IJmond y del movimiento de sacerdotes contestatarios de la Septuaginta, no puede verse, lógicamente, como una consecuencia del Concilio. Los teóricos de esta radicalización social y política forman un grupo restringido, pero muy mediatizado, de intelectuales que trabajan para conciliar el cristianismo y el marxismo ${ }^{24}$. A sus ojos, el marxismo es una especie de mesianismo secularizado, anclado en la tradición judeo-cristiana. Estos adoptaban una interpretación materialista de la Biblia: la exégesis debía obrarse ayudada por las categorías propias del pensamiento marxista. Formados, la mayor parte de ellos, en la «teología política» de Juan Bautista Metz, en Münster, ocupaban las redacciones de los periódicos progresistas más influyentes, como Te Elfder Ure (católico) o Wending (protestante). En esos medios encuentran un lugar privilegiado para difundir sus ideas. Es así como la Universidad de Utrecht lanzó una formación teológica y socio-educativa en colaboración con la Academia social De Horst, muy escorada hacia la izquierda, con el fin de formar a los futuros animadores parroquiales. Esta formación, que no era corriente en el seno de la Iglesia, existió hasta 1982. Pero la audiencia de la teología crítica disminuirá progresivamente, hasta el golpe de gracia que supondrá la caída del muro de Berlín en 1989.

24 Véase Theo SaleminK, Meervoudige modernisering. Linkse katholieken in de lange jaren zestig, en Contrapunten tussen radicaal christendom, restauratie en pluralisering, Valkhof Pers, Nijmegen, 2010, pp. 50-78. 


\section{MEDIACIÓN INTERNACIONAL}

La experiencia holandesa ha tenido un gran eco internacional. Los reportajes han aparecido no solo en Paris Match, sino también en Epoca y Panorama (Italia), De Standaard (Bélgica), Time Magazine, el National Catholic Reporter y el New York Times (Estados Unidos). Esta mediación ha sido llevada a cabo, entre 1968 y 1972, por parte de los sacerdotes contestatarios de la Septuaginta, sin lugar a dudas el grupo más radical de la época.

\section{Septuaginta}

En sus inicios, se trataba de un grupo de alrededor de setenta sacerdotes que se reunieron por primera vez con ocasión del matrimonio de Jos Vrijburg, capellán de la Parroquia estudiantil de Ámsterdam ${ }^{25}$. Su presidente era Jan Ruijter, que celebraba los domingos en Beverwijk, en la comunidad originaria de lo que sería años más tarde la Parroquia crítica de IJmond. La Septuaginta comenzó por enviar una carta a los obispos de los Países Bajos pidiendo que, a título experimental, la misa pudiera ser dicha por sacerdotes casados en un cierto número de parroquias. La supresión de la obligación del celibato para los sacerdotes era, entonces, uno de sus objetivos principales, evidentemente, en vano. Menos de un año más tarde, la Septuaginta envió otra carta, esta vez a los sacerdotes de los Países Bajos, explicando que su acción no se limitaba solo a esta cuestión. Se trataba, con más amplitud, de liberar a la Iglesia «de su inercia, su autoritarismo y su alianza con las fuerzas políticas y económicas establecidas, [...] con el fin de asegurar su credibilidad para anunciar la Buena Nueva $»^{26}$. De hecho, desde ese momento, la Septuaginta intentó reformar la sociedad modernizando la Iglesia.

A principios de 1969, la Septuaginta organizó en Ámsterdam una segunda reunión, de gran envergadura, en la que participaron representantes de grupos de sacerdotes contestatarios alemanes (Arbeitsgemeinschaften von Priestergruppen) y franceses (Échanges et Dialogue). Una de las decisiones tomadas en esa ocasión

25 Sobre la Septuaginta y su acción internacional, véase Leo LAEYENDECKER, «Septuagint». De lotgevallen van een vernieuwingsbeweging, en Osmund SCHREUDER, Leo VAN SNIPPENBURG (dirs.), Religie in de Nederlandse samenleving. De vergeten factor, Ambo, Baarn, 1990, pp. 90-117; Chris DoLS, Benjamin ZIEMANN, Progressive Participation and Transnational Activism in the Catholic Church after Vatican II: The Dutch and West German Examples, en Fournal of Contemporary History, 50 (2015), pp. 465-485.

26 Citado por LaEYENDECKER, Septuagint, op. cit., p. 91. 
fue la de hacerse presente en el sínodo de obispos europeos previsto, un poco más tarde, en ese mismo año en Chur, Suiza. Allí se encuentran las delegaciones de los grupos presentes en Ámsterdam, pero también Présence et Témoignage (Bélgica) y el Solidaritätsgruppe de Viena (Austria) ${ }^{27}$. No hubo diálogo con los obispos, pero la prensa, ampliamente representada, dio una gran cobertura a este encuentro. Los líderes de la Septuaginta declararon que los sacerdotes debían apartarse masivamente de «aquellos que mantenían el sistema de opresión y que era necesario denunciar a la Iglesia cuando ella callaba ante la injusticia, y se comprometía y compartía los objetivos de los poderes establecidos ${ }^{28}$.

Ese mismo año, en octubre, un sínodo de los obispos de todo el mundo tuvo lugar en Roma, lo que le dio a la Septuaginta la oportunidad de manifestarse de nuevo. Un detalle revelador del clima de la época fue que los sacerdotes contestatarios no encontraron ningún lugar para reunirse por parte de las instituciones eclesiásticas siendo alojados por el seminario valdense. Consciente de la inutilidad de cualquier tipo de lobby frente a los padres sinodales, los sacerdotes contestatarios discutieron entre ellos sobre el «pequeño libro rojo», un conjunto de textos compuesto por la Septuaginta titulado Die Kirche befreien für die Befreiung der Welt (Liberar la Iglesia para liberar al mundo). Pero las posiciones radicales de la Septuaginta no eran sostenidas unánimemente.

Como hemos visto, la Septuaginta se había fundado sobre la oposición a la obligación del celibato eclesiástico, pero el Vaticano se opuso sistemáticamente a entrar a ese debate, cosa que el arzobispo de los Países Bajos, el cardenal Bernard Alfrink, imploraba durante una de sus visitas a Roma en 1970. El grupo se vio obligado a abandonar, provisionalmente, esta reivindicación, volcándose en las parroquias críticas y en las comunidades de base. Pero al tratar de imponerles una línea de conducta, la Septuaginta hizo una demostración clara de clericalismo que lo alineaba con la tendencia que pretendía denunciar. En 1970, el tercer encuentro de la Asamblea europea de sacerdotes tuvo lugar en Ámsterdam. Esta reunión estuvo marcada por representantes del tercer mundo, venidos sobretodo de Sudamérica, erigiéndose en los protagonistas del encuentro. La teología de la liberación, inspirada en el marxismo, parecía abrir nuevos horizontes. Además, se organizaron manifestaciones pidiendo la independencia «inmediata» para Surinam y las Antillas holandesas.

Con motivo de las elecciones de 1971 en los Países Bajos, la Septuaginta sugirió al Centro de acción ecuménica la firma de un manifiesto común. La re-

27 Septuagintgroep, Septuagint van Chur naar Rome. Dossier van solidaire priestergroepen, De Horstink, Amersfoort, 1969.

28 Citado por LAEYENDECKER, Septuagint, op. cit., p. 97. 
acción dolida de Daniel de Lange, director del Centro de acción ecuménica, era muestra de la marginalización de la Septuaginta. De Lange les aconsejó «continuar su trabajo para lograr la evolución de la opinión dentro de la Iglesia y abstenerse de posturas radicales en el ámbito político, porque si no se arriesgaban a recibir críticas desde todas partes ${ }^{29}$. Pero la huida hacia delante de la Septuaginta tiene su origen en las tensiones internas que minaban el grupo y eran causadas por serias «divergencias ideológicas». Al final, según el sociólogo Laeyendecker, no quedó más que un «pequeño grupo que se contentaba con discutir internamente y que no contaba, desde hacía tiempo, con el apoyo popular» ${ }^{30}$. Cuando la Septuaginta desaparece en 1975, sus miembros se afiliaron a otras organizaciones o se integraron en las comunidades de base.

\section{EL PAPEL DE LOS MEDIOS}

La imagen de los católicos de los años 60 que predomina hasta el día de hoy en la historiografía del catolicismo holandés ha sido conformada, como lo demuestra Marjet Derks, por los medios internacionales. Como era en los Países Bajos donde las transformaciones de la sociedad eran más espectaculares, los periodistas de la prensa extranjera seguían con avidez todas las novedades y todos los conflictos. Sus artículos eran, a su vez, rápidamente reproducidos, por los periódicos holandeses, creando un fenómeno curioso de «percepción de sí mismo a través de los ojos de otros» ${ }^{31}$.

Es necesario añadir que los protagonistas de los hechos eran poco discretos. Los grupos más críticos, en particular, no dudaban en explicar con detalle en sus comunicados a la prensa sus decisiones, por ejemplo, en el caso de la celebración de las misas por sacerdotes casados. Igualmente, daban noticia del contenido de sus reuniones con la jerarquía episcopal ${ }^{32}$, para meterles presión frente a la opi-

29 Ibid., p. 107.

30 Ibid., p. 114.

31 Véase Marjet DERKS, Een andere tijdgeest. Conservatieven, «normaal-katholieken» en het dominante beeld van vernieuwing in postconciliair Nederland (1962-1985), en Peter VAN DAM, James KENNEDY, Friso WIELINGA (dir.), Achter de zuilen. Op zoek naar religie in naoorlogs Nederland, Amsterdam, Amsterdam University Press, 2014, pp. 201-230; id., Media, Gender and the Invisible Conservative Dutch Catholic in the long 1960s, en Schweizerische Zeitschrift für Religions-und Kulturgeschichte, 104 (2010), pp. 135-154.

32 Véase, por ejemplo, la entrevista entre la Parroquia crítica IJmond y el obispo de Haarlem en 1972, en AUWERDA, Kerk van onderen, op. cit., pp. 13-25. 
nión pública, con el riesgo de que esa estrategia se volviera en su contra: cuando se hizo pública la decisión del cardenal Alfrink de rechazar la participación de los pastores protestantes en las eucaristías de la Parroquia estudiantil de Utrecht, al obispo de Rotterdam no le quedó otro remedio que prohibir a su vez las de la Parroquia estudiantil de Leyde, cuando hasta ese momento había mirado hacia otro lado.

La crisis petrolera de 1973 produjo un desencanto brutal y modificó profundamente el estado de ánimo de la sociedad holandesa, desde ese momento bajada a la tierra por las realidades económicas. Paralelamente, el endurecimiento de la Santa Sede, la recuperación del control por parte de los episcopados nacionales y el triunfo del catolicismo conservador dificultaban el camino de las corrientes progresistas $^{33}$. El catolicismo holandés quedó marcado durante decenios por el abismo que separaba a conservadores de progresistas. Aún en retirada, estos últimos sin embargo han logrado que se continuara hablando de ellos. En 1972, crearon el movimiento «Iglesia abierta», como reacción al nombramiento de Joannes Gijsen como obispo de Roermond, pero esta iniciativa no tuvo un largo recorrido debido a la falta de organización. En 1984, la Asociación Mariënburg, fundada como respuesta al manifiesto titulado «Testimonios del Espíritu que habita en nosotros» (1983), tuvo un poco más de éxito y aún continúa en activo, a pesar del envejecimiento de sus miembros y el declinar de sus efectivos. Finalmente, después de la visita de Juan Pablo II a los Países Bajos en 1985, los progresistas se reunieron para alumbrar el Movimiento del 8 de mayo, que congregaba a unos 10000 participantes en sus primeras asambleas anuales. Las relaciones de este movimiento contestatario con el episcopado han sido muy tensas. A pesar de los intercambios con algún que otro prelado y la participación de dos de ellos «a título personal» en el encuentro de 1995, ninguna entente ha sido posible. Frente a la pérdida de efectivos, el Movimiento del 8 de mayo prefirió disolverse en el año $2003^{34}$. La epopeya progresista, a veces demasiado escorada a la izquierda, no había durado más que una decena de años.

33 Para lo que sigue, véase Lodewijk WINKELER, Stromingen in katholiek Nederland, Kok, Kampen, 2004.

34 Tom van Den Beld, Het andere gezicht van de kerk. De Acht Mei Beweging 1985-2003, Valkhof Pers, Nijmegen, 2015. 